\title{
Decay width and coupling constants of charm and bottom mesons
}

\author{
Pallavi Gupta*i \\ School of Physics and Material Science, Thapar University, Patiala \\ E-mail: 10 gupta.pallaviegmail.com
}

\author{
Alka Upadhyay \\ School of Physics and Material Science, Thapar University, Patiala \\ E-mail: alka.iisc@gmail.com
}

In the last decade, charmed and bottom meson spectroscopy have seen great success in experimental sector. Experiments like LHCb, Babar etc are providing many new states which are being added to their spectroscopy. Newly predicted states like B(5970), $D_{2}(3000), D^{*}(3000), B(5840)$ and many more still need to be assigned their proper place in the spectroscopy. So we studied the decay constant and the coupling constants of these states using the heavy quark effective theory as our model. We analyzed the two-body strong decays of the above states to their ground state mesons with light pseudo-scalar mesons $(\pi, \eta, K)$. We also obtained the ratios among their strong decays, which can be confronted to the experimental data for the verification of their $J^{P}$ states. In addition to this, we also study the strong decays of their spin and strange partners, which are still experimentally not observed, and may be useful for future experiments in searching for these heavy-light mesons.

XVII International Conference on Hadron Spectroscopy and Structure - Hadron2017

25-29 September, 2017

University of Salamanca, Salamanca, Spain

*Speaker.

${ }^{\dagger}$ A footnote may follow. 


\section{Introduction}

In the past decade, many new discoveries have filled the spectroscopy of charm and bottom mesons. Recently in 2016, LHCb collaboration have used the Dalitz plot analysis to study the resonant substructures $B^{-} \rightarrow D^{+} \pi^{-} \pi^{-}$decays in the pp collision at a center-of-mass energy 7 $\mathrm{TeV}$ and observed a new charm resonance $D_{2}^{*}(3000)$ with mass $M=3214 \pm 29 \mathrm{MeV}$ and decay width $\Gamma=186 \pm 38 \mathrm{MeV}$ [1]. In 2013, LHCb has also observed charm resonance $D_{J}(3000)^{0}$ in the $D^{*+} \pi^{-}$final state and $D_{J}^{*}(3000)^{+}$and $D_{J}^{*}(3000)^{0}$ in the $D^{0} \pi^{+}$and $D^{+} \pi^{-}$mass spectra respectively [2]. In the bottom sector, two new resonances $B(5970)^{+}$and $B(5970)^{0}$ are observed by the CDF collaboration in 2013 [3]. Masses and decay widths of these resonances are: $M\left(B(5970)^{+}\right)=$ $5961 \pm 13 \mathrm{MeV}$ with decay width $\Gamma\left(B(5970)^{+}\right)=60 \pm 40 \mathrm{MeV}$ and mass $M\left(B(5970)^{0}\right)=5977 \pm$ $13 \mathrm{MeV}$ and width $\Gamma\left(B(5970)^{0}\right)=70 \pm 30 \mathrm{MeV}$. Also in 2015 , LHCb also predicted a bottom resonance $B_{J}^{+, 0}(5840)$ with mass $M=5862.90 \pm 5 \mathrm{MeV}$ and decay width $\Gamma=127.40 \pm 16.70$ $\mathrm{MeV}$ [4]. The $J^{P^{\prime}} s$ of these newly observed states are yet to be confirmed. It is very important to assign a proper $J^{P}$ to these states, as large amount of information like decay width, branching ratios, strong coupling etc are based on their $J^{P}$. Theoretically, various models like quark pair creation model, heavy quark effective theory, QCD sum rules etc are available to study the heavy-light system. Various theoretical models suggests different $J^{P}$ states to these mesons as these models uses different parameters. Authors in [5] suggests $D_{J}^{*}(3000)$ charm meson to belong to either $1 F_{\frac{5}{2}} 2^{+}$or $1 F_{\frac{7}{2}} 4^{+}$state and $D_{J}(3000)$ either to $1 F_{\frac{7}{2}} 3^{+}$or $2 P_{\frac{1}{2}} 1^{+}$state. But Ref.[6] have suggested various other possibilities for their $J^{P^{\prime}} s$ and concluded $2 P\left(0^{+}, 1^{+}\right)$to be the most favorable $J^{P}$ of $\left(D_{J}^{*}(3000)\right),\left(D_{J}(3000)\right)$ by studying their branching ratio. ${ }^{3} P_{0}$ model suggested the $J^{P}$ of the charm state $D_{2}^{*}(3000)$ to be $3 P_{\frac{3}{2}} 2^{+}$state, but the other possibility like $2 F_{\frac{5}{2}} 2^{+}$may not be completely excluded [7]. Using the effective lagrangian approach, in our paper [8] we suggested $D_{2}^{*}(3000)$ to belong to $1 F\left(2^{+}\right)$state. Similarly for the bottom sector, many theorists suggest $B_{J}^{*}(5970)$ state to be in $\left.2 S_{1 / 2}\left(1^{-}\right)\right)$state $[9,10]$. Li-Ye Xio using chiral quark model suggested it to be in $1 D_{5 / 2}\left(3^{-}\right)$ state [11]. And the $B_{J}(5840)$ state is suggested to belong to the $B\left(2 S_{1 / 2}\left(0^{-}\right)\right)$state in the bottom spectra [12]. In this paper, we study these newly observed charm and bottom states using the effective lagrangian approach and calculate their branching ratios and the strong coupling constants involved. At the leading order approximation, the mass and the spin degeneracy of heavy hadrons appears because of approximate internal symmetry of the lagrangian. Along with these states, we also predict the decay width and the branching ratios for the spin and the strange partners of these sates, which are theoretically known but experimentally unobserved. The paper is arranged as follow: a brief review of HQET is given in section 2. The section 3 gives the study of $D_{J}(3000)$, $D_{J}^{*}(3000), D_{2}^{*}(3000), B_{J}(5840)$ and $B_{J}^{*}(5970)$ and their spin and strange partner states, followed by conclusion in section 4 .

\section{Framework}

Heavy quark effective theory is an effective QCD theory for $N_{f}$ heavy quarks with their four velocity fixed. In this theory, spin and parity of the heavy quark decouples from the light degrees of freedom as they interact through the exchange of soft gluons. Heavy mesons are classified in doublets in relation to the total conserved angular momentum i.e. $s_{l}=s_{\bar{q}}+l$, where $s_{\bar{q}}$ and $l$ are 
the spin and orbital angular momentum of the light degree of freedom respectively. For $l=0$ (S-wave) the doublet is represented by $\left(P, P^{*}\right)$ with $J_{s_{l}}^{P}=\left(0^{-}, 1^{-}\right)_{\frac{1}{2}}$, which for $l=1$ (P-wave), there are two doublets represented by $\left(P_{0}^{*}, P_{1}^{\prime}\right)$ and $\left(P_{1}, P_{2}^{*}\right)$ with $J_{s_{l}}^{P}=\left(0^{+}, 1^{+}\right)_{\frac{1}{2}}$ and $\left(1^{+}, 2^{+}\right)_{\frac{3}{2}}$ respectively. Two doublets of $l=2$ (D-wave) are represented by $\left(P_{1}^{*}, P_{2}\right)$ and $\left(P_{2}^{\prime}, P_{3}^{*}\right)$ belonging to $J_{s_{l}}^{P}=\left(1^{-}, 2^{-}\right)_{\frac{3}{2}}$ and $\left(2^{-}, 3^{-}\right)_{\frac{5}{2}}$ respectively. These doublets are described by the effective superfield $H_{a}, S_{a}, T_{a}, X_{a}, Y_{a}$, where the field $H_{a}$ describe the $\left(P, P^{*}\right)$ doublet i.e. S-wave, $S_{a}$ and $T_{a}$ fields represents the P-wave doublets $\left(0^{+}, 1^{+}\right)_{\frac{1}{2}}$ and $\left(1^{+}, 2^{+}\right)_{\frac{3}{2}}$ respectively [13, 9]. Using the chiral lagrangian terms $L_{H H}, L_{S H}, L_{T H}, L_{Y H}, L_{Z H}$, the two body strong decays of $Q \bar{q}$ system to final state light pseudo-scalar mesons $\mathrm{M}(\pi, \eta, K)$ are given as $\left(0^{-}, 1^{-}\right) \rightarrow\left(0^{-}, 1^{-}\right)+M$

$$
\begin{aligned}
& \Gamma\left(1^{-} \rightarrow 1^{-}\right)=C_{M} \frac{g_{H H}^{2} M_{f} p_{M}^{3}}{3 \pi f_{\pi}^{2} M_{i}} \\
& \Gamma\left(1^{-} \rightarrow 0^{-}\right)=C_{M} \frac{g_{H H}^{2} M_{f} p_{M}^{3}}{6 \pi f_{\pi}^{2} M_{i}} \\
& \Gamma\left(0^{-} \rightarrow 1^{-}\right)=C_{M} \frac{g_{H H}^{2} M_{f} p_{M}^{3}}{2 \pi f_{\pi}^{2} M_{i}}
\end{aligned}
$$

$\left(0^{+}, 1^{+}\right) \rightarrow\left(0^{-}, 1^{-}\right)+M$

$$
\begin{aligned}
& \Gamma\left(1^{+} \rightarrow 1^{-}\right)=C_{M} \frac{g_{S H}^{2} M_{f}\left(p_{M}^{2}+m_{M}^{2}\right) p_{M}}{2 \pi f_{\pi}^{2} M_{i}} \\
& \Gamma\left(0^{+} \rightarrow 0^{-}\right)=C_{M} \frac{g_{S H}^{2} M_{f}\left(p_{M}^{2}+m_{M}^{2}\right) p_{M}}{2 \pi f_{\pi}^{2} M_{i}}
\end{aligned}
$$

$\left(2^{+}, 3^{+}\right) \rightarrow\left(0^{-}, 1^{-}\right)+M$

$$
\begin{aligned}
& \Gamma\left(2^{+} \rightarrow 1^{-}\right)=C_{M} \frac{8 g_{Z H}^{2}}{75 \pi f_{\pi}^{2} \Lambda^{4}} \frac{M_{f}}{M_{i}}\left[p_{M}^{5}\left(m_{M}^{2}+p_{M}^{2}\right)\right] \\
& \Gamma\left(2^{+} \rightarrow 0^{-}\right)=C_{M} \frac{4 g_{Z H}^{2}}{25 \pi f_{\pi}^{2} \Lambda^{4}} \frac{M_{f}}{M_{i}}\left[p_{M}^{5}\left(m_{M}^{2}+p_{M}^{2}\right)\right] \\
& \Gamma\left(3^{+} \rightarrow 1^{-}\right)=C_{M} \frac{4 g_{Z H}^{2}}{25 \pi f_{\pi}^{2} \Lambda^{4}} \frac{M_{f}}{M_{i}}\left[p_{M}^{5}\left(m_{M}^{2}+p_{M}^{2}\right)\right]
\end{aligned}
$$

In the above expressions of decay widths, $M_{i}$ and $M_{f}$ stands for initial and final meson mass. All hadronic coupling constants depends on the radial quantum number. For the decay within $\mathrm{n}=1$ they are notated as $g_{H H}, g_{S H}$ etc, and the decay from $\mathrm{n}=2$ to $\mathrm{n}=1$ they are represented by $\widetilde{g}_{H H}^{2}, \widetilde{g}_{S H}^{2}$. $p_{M}$ and $m_{M}$ are the final momentum and mass of the light pseudo-scalar meson. The coefficient $C_{\pi^{ \pm}}, C_{K^{ \pm}}, C_{K^{0}}, C_{\bar{K}^{0}}=1, C_{\pi^{0}}=\frac{1}{2}$ and $C_{\eta}=\frac{1}{6}$ or $\frac{2}{3}$. Different values of $C_{\eta}$ corresponds to the initial state being $c \bar{u}, c \bar{d}$ or $c \bar{s}$ respectively. Higher order terms of spin and flavor violation corrections which are of order $\frac{1}{m_{Q}}$ are neglected in these equations to avoid the admittance of new unknown coupling constants. These unknown coupling constants can either be theoretically predicted or can be determined indirectly from the known experimental values of the decay widths. 


\section{Numerical Analysis}

Assigning a proper $J^{P}$ to the newly observed heavy-light mesons is very crucial, as it helps in retrieving many properties like decay widths, strong coupling constants, branching ratios etc. From the available theoretical and experimental information, we identify the $D_{J}(3000), D_{J}^{*}(3000)$, $D_{2}^{*}(3000), B_{J}(5840)$ and $B_{J}^{*}(5970)$ states as

$$
\begin{aligned}
\left(D_{J}(3000), D_{J}^{*}(3000)\right)=\left(0^{+}, 1^{+}\right)_{\frac{1}{2}} & \text { with } & n=2, L=1 \\
D_{2}^{*}(3000)=\left(2^{+}\right)_{\frac{5}{2}} & \text { with } & n=1, L=3 \\
\left(B_{J}(5840), B_{J}^{*}(5970)\right)=\left(0^{-}, 1^{-}\right)_{\frac{1}{2}} & \text { with } & n=2, L=0
\end{aligned}
$$

The numerical value for the partial decay widths and the branching ratios of these states are listed in Table 1 and Table 2. We also equate the experimental decay widths and obtain the strong coupling constants $\widetilde{g}_{H H}, \widetilde{g}_{S H}$ and $g_{Z H}$ as $\widetilde{g}_{H H}=0.17, \widetilde{g}_{S H}=0.12$ and $g_{Z H}=0.15$. For this, we have neglected the large value of $\widetilde{g}_{H H}=0.49$, in comparison with its previous theoretical values [13]. Until now, the experimental information on the strong decay widths for $D_{s}\left(2^{3} P_{0}\right), D_{s}\left(2^{1} P_{1}\right), D_{s}\left(1^{3} F_{2}\right)$, $D_{s}\left(1^{1} F_{3}\right), D\left(1^{1} F_{3}\right), B_{s}\left(2^{1} S_{0}\right)$ and $B_{s}\left(2^{3} S_{1}\right)$ states is unavailable. So in this paper, we calculated the partial decay widths and the branching ratios of the above states which will be helpful for the future experimental study of these states. Masses for these states are used as $M\left(D_{s}\left(2^{3} P_{0}\right)\right)=3054 \mathrm{MeV}$, $M\left(D_{s}\left(2^{1} P_{1}\right)\right)=3154 \mathrm{MeV}, M\left(D_{s}\left(1^{3} F_{2}\right)\right)=3266 \mathrm{MeV}, M\left(D_{s}\left(1^{1} F_{3}\right)\right)=3230 \mathrm{MeV}, M\left(D\left(1^{1} F_{3}\right)\right)=$ $3145 \mathrm{MeV}, M\left(B_{s}\left(2^{1} S_{0}\right)\right)=5976 \mathrm{MeV}$ and $M\left(B_{s}\left(2^{3} S_{1}\right)\right)=5992 \mathrm{MeV}$ [14]. The partial widths and the branching ratios of the above states are listed in Table 1 and Table 2. By using the value of the coupling constant obtained above, the total decay widths of these states comes as $\Gamma\left(D_{s}\left(2^{3} P_{0}\right)\right)=$ 212.92 MeV, $\Gamma\left(D_{s}\left(2^{1} P_{1}\right)\right)=205.78 \mathrm{MeV}, \Gamma\left(D_{s}\left(1^{3} F_{2}\right)\right)=187.10 \mathrm{MeV}, \Gamma\left(D_{s}\left(1^{1} F_{3}\right)=150.61 \mathrm{MeV}\right.$, $\Gamma\left(D\left(1^{1} F_{3}\right)=82.62 \mathrm{MeV}, \Gamma\left(B_{s}\left(2^{1} S_{0}\right)\right)=32.47 \mathrm{MeV}\right.$ and $\Gamma\left(B_{s}\left(2^{3} S_{1}\right)=42.75 \mathrm{MeV}\right.$. The partial decay widths predicted in this paper are comparable with the values predicted in Ref.[15]. Ratio in Table 1 and Table 2 represents $\widehat{\Gamma}=\frac{\Gamma}{\left.\Gamma\left(P_{J}^{*}\right) \rightarrow P^{*+} \pi^{-}\right)}$for non-strange states and $\widehat{\Gamma}=\frac{\Gamma}{\Gamma\left(P_{s J}^{*} \rightarrow P^{* 0} K^{+}\right)}$for strange states except for $2 P_{1 / 2} 0^{+}$and $2 P_{s 1 / 2} 0^{+}$states. Here $\mathrm{P}$ can be D or B. For $2 P_{1 / 2} 0^{+}$state, ratio is given as $\widehat{\Gamma}=\frac{\Gamma}{\left.\Gamma\left(B_{J}^{*}\right) \rightarrow B^{+} \pi^{-}\right)}$and for $2 P_{s 1 / 2} 0^{+}$it is $\widehat{\Gamma}=\frac{\Gamma}{\Gamma\left(B_{s J}^{*} \rightarrow B^{+} K^{-}\right)}$.

\section{Conclusion}

In the paper, we have examined the heavy-light states $D_{J}(3000), D_{J}^{*}(3000), D_{2}^{*}(3000), B_{J}(5840)$ and $B_{J}^{*}(5970)$ with $J^{P}$ states $2 P_{\frac{1}{2}} 0^{+}, 2 P_{\frac{1}{2}} 1^{+}, 1 F_{\frac{5}{2}} 2^{+}, 2 S_{\frac{1}{2}} 0^{-}$and $2 S_{\frac{1}{2}} 1^{-}$respectively. Here we have used the HQET lagrangian at the leading order approximation, and studied their two body strong decay behavior with the emission of light pseudo-scalar mesons $(\pi, \eta, K)$. We have computed the branching ratios and the coupling constants $\widetilde{g}_{H H}, \widetilde{g}_{S H}$ and $g_{Z H}$ for the above states that can be useful for the future experimental data to compare with.

The obtained coupling constant helps in calculating the strong decay channels for the experimentally missing $D_{s}\left(2^{3} P_{0}\right), D_{s}\left(2^{1} P_{1}\right), D_{s}\left(1^{3} F_{2}\right), D_{s}\left(1^{1} F_{3}\right), D\left(1^{1} F_{3}\right), B_{s}\left(2^{1} S_{0}\right), B_{s}\left(2^{3} S_{1}\right)$ states. Thus calculation of these decay widths will be able to open a window to investigate the higher excitations of charm mesons at the LHCb, BaBar, BESIII. 


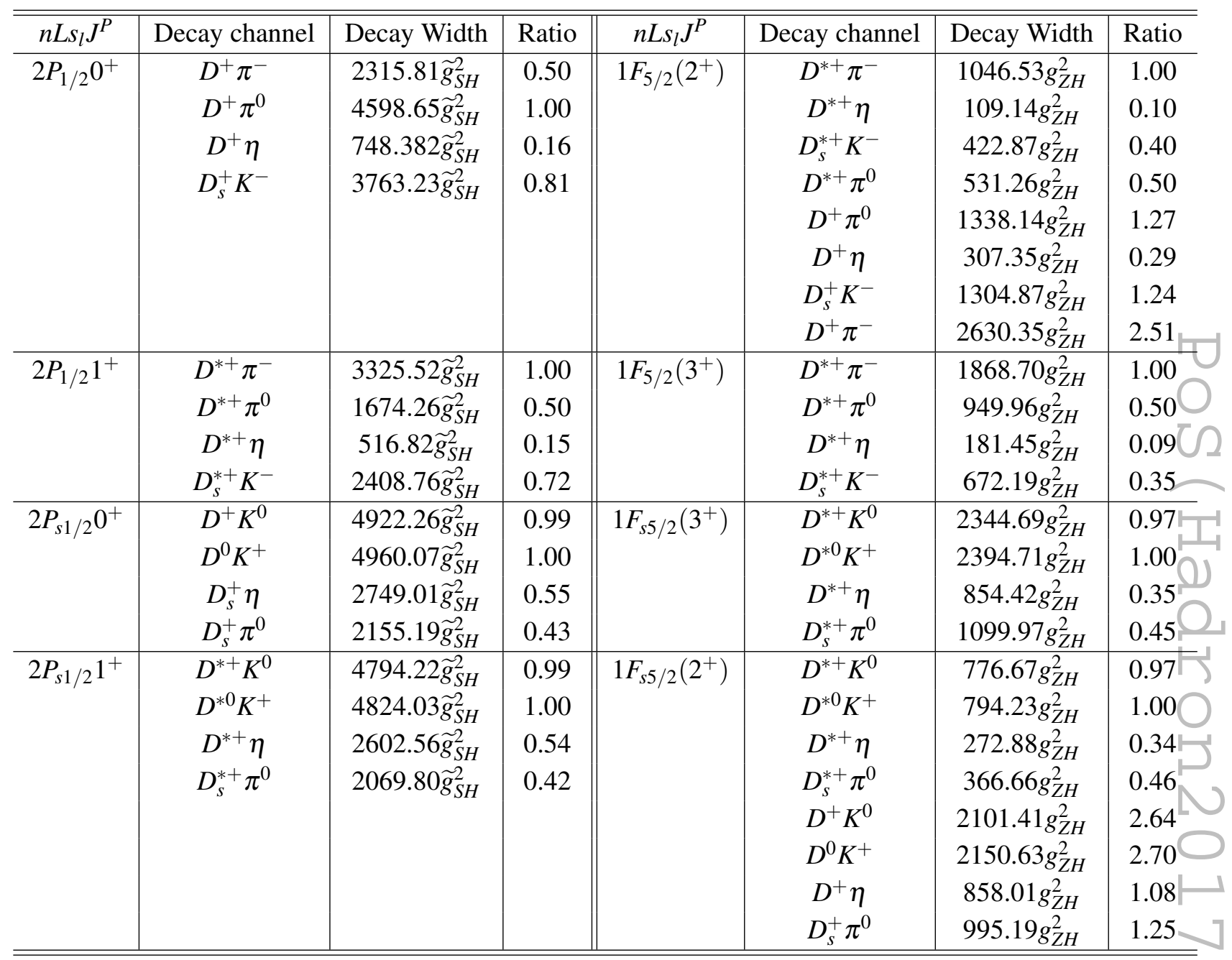

Table 1: Partial decay width and the branching ratios for the $2 \mathrm{P}$ and $1 \mathrm{~F}$ charm meson family. The values of the decay widths are in $\mathrm{MeV}$ units.

\begin{tabular}{c|c|c|c|c|c|c}
\hline$n L s_{l} J^{P}$ & \multicolumn{3}{|c|}{ Non-Strange Mesons } & \multicolumn{3}{c|}{ Strange Mesons } \\
\cline { 2 - 7 } & Decay channel & Decay Width & Ratio & Decay channel & Decay Width & Ratio \\
\hline \multirow{3}{*}{$2 S_{1 / 2} 0^{-}$} & $B^{* 0} \pi^{0}$ & $523.71 \widetilde{g}_{H H}^{2}$ & 1.00 & $B^{*} K^{0}$ & $521.96 \widetilde{g}_{H H}^{2}$ & 1.00 \\
& $B^{* 0} \eta$ & - & - & $B_{s}^{*} \pi^{0}$ & $593.72 \widetilde{g}_{H H}^{2}$ & 1.13 \\
& $B_{s}^{*} K^{0}$ & - & - & $B_{s}^{*} \eta$ & $7.85 \widetilde{g}_{H H}^{2}$ & 0.01 \\
\hline \multirow{5}{*}{$2 S_{1 / 2} 1^{-}$} & $B^{0} \pi^{0}$ & $370.76 \widetilde{g}_{H H}^{2}$ & 0.60 & $B^{0} K^{0}$ & $304.08 \widetilde{g}_{H H}^{2}$ & 0.74 \\
& $B^{+} \pi^{-}$ & $739.50 \widetilde{g}_{H H}^{2}$ & 1.20 & $B^{+} K^{-}$ & $311.74 \widetilde{g}_{H H}^{2}$ & 0.76 \\
& $B^{0} \eta$ & $30.90 \widetilde{g}_{H H}^{2}$ & 0.05 & $B_{s} \pi^{0}$ & $272.32 \widetilde{g}_{H H}^{2}$ & 0.66 \\
& $B_{S} K^{0}$ & $105.94 \widetilde{g}_{H H}^{2}$ & 0.17 & $B_{s} \eta$ & $43.51 \widetilde{g}_{H H}^{2}$ & 0.10 \\
& $B^{*} \pi^{0}$ & $612.79 \widetilde{g}_{H H}^{2}$ & 1.00 & $B^{*} K^{0}$ & $409.71 \widetilde{g}_{H H}^{2}$ & 1.00 \\
& $B^{*} \eta$ & $34.57 \widetilde{g}_{H H}^{2}$ & 0.05 & $B_{s}^{*} \pi^{0}$ & $430.96 \widetilde{g}_{H H}^{2}$ & 1.05 \\
& $B_{s}^{*} K^{0}$ & $85.79 \widetilde{g}_{H H}^{2}$ & 0.13 & $B_{s}^{*} \eta$ & $18.89 \widetilde{g}_{H H}^{2}$ & 0.04 \\
\hline
\end{tabular}

Table 2: Partial decay width and the branching ratios for the $2 \mathrm{~S}$ bottom meson family. The values of the decay widths are in $\mathrm{MeV}$ units. 


\section{Acknowledgement}

The authors gratefully acknowledge the financial support by the Department of Science and Technology (SB/FTP/PS-037/2014), New Delhi.

\section{References}

[1] R. Aaij et al [LHCb Collaboration], Amplitude analysis of $B^{-} \rightarrow D^{+} \pi^{-} \pi^{-}$decays, Phys. Rev. D, 94 072001 (2016).

[2] R. Aaij et al [LHCb Collaboration], Study of $D_{J}$ meson decays to $D^{+} \pi^{-}, D^{0} \pi^{+}$and $D^{*+} \pi^{-}$final states in pp collisions, JHEP 1451309 (2013).

[3] T. Aaltonen et. al., Study of orbitally excited B mesons and evidence for a new B $\pi$ resonance, Phys. Rev. D 90012013 (2014).

[4] R.aij et. al. [LHCb collaboration] Precise measurements of the properties of the $B_{1}(5721)^{0,+}$ and $B_{2}^{*}(5747)^{0,+}$ states and observation of $B^{+, 0} \pi^{-,+}$mass structures, JHEP 0241504 (2015).

[5] Guo Liang Yu et. al., Systematic analysis of the $\left.D_{J}(2580), D_{J}^{*} 2650\right), D_{J}(2740), D_{J}^{*}(2760), D_{J}(3000)$ and $D_{J}^{*}(3000)$ in D meson family, Chin.Phys. C, 39, 063101 (2015).

[6] Meenakshi Batra, Alka Upadhyay, Strong Decay Widths and Coupling Constant of Recent Charm Meson States, Eur.Phys.J. C, 75319 (2015).

[7] Jun-Zhang Wang et. al., Revealing the inner structure of the newly observed $D_{2}^{*}(3000)$, Phys. Rev. D, 94, 094044 (2016).

[8] P. Gupta et.al., Analysis of strong decays of charmed mesons $D_{2}^{*}(2460), D_{0}(2560), D_{2}(2740)$, $D_{1}(3000), D_{2}^{*}(3000)$ and their spin partners $D_{1}^{*}(2680), D_{3}^{*}(2760)$ and $D_{0}^{*}(3000)$, submitted in Phys. Rev. D (2017).

[9] Z.G. Wang, Strong decays of the bottom mesons $B_{1}(5721), B_{2}(5747) B 2, B_{s 1}(5830), B_{s 2}(5840)$ and B(5970), Eur. Phys. J. Plus, 129186 (2014).

[10] Hao Xu et.al., Newly observed B(5970) and the predictions of its spin and strange partners, Phys. Rev. D 89, 097502 (2014).

[11] Li-Ye Xiao et. al., Strong decays of higher excited heavy-light mesons in a chiral quark model, Phys. Rev. D, 90074029 (2014).

[12] Q. F. Lu et. al., Excited bottom and bottom-strange mesons in the quark model, Phys. Rev. D, 94, 074012 (2016).

[13] P. Colangelo et.al., New meson spectroscopy with open charm and beauty, Phys. Rev. D, 86054024 (2012).

[14] D. Ebert et. al., Heavy-light meson spectroscopy and Regge trajectories inătheărelativistic quark model, Eur. Phys. J. C, 66197 (2010).

[15] Q.T. Song et. al., Higher radial and orbital excitations in the charmed meson family, Phys. Rev. D, 92 074011 (2015). 